\title{
Construction mode of safety engineering laboratory
}

\author{
Kaiwu Liang, Zhiyong Cai, Chun Liu, Wenhe Wang \\ Safety Engineering Institute, Chong Qing University of Science \& Technology, Chongqing, China \\ email: 578287768@qq.com
}

Keywords: component; integrate resources; highlighting characteristics; safety engineering lab

\begin{abstract}
In view of the central and local project-the construction of safety engineering lab, the paper put forward the safety engineering lab construction mode-integrate resources and highlighting characteristics based on the training objectives and teaching requirements of safety engineering discipline and combined with the pre-construction actual. In this way the complete safety engineering experimental system could be formed and the characteristics of safety inspection and safety assessment was highlighted. The related problems in the development of safety engineering laboratory were solved through the practice.
\end{abstract}

Since the 21 st century, the special advantage discipline laboratory project of university which was built by central and local governments, to a certain extent, solved some problems such as the university laboratory construction funds shortage, the lower resource utilization, no outstanding features, etc. It promoted the development of the university laboratory and the combination of laboratory teaching and scientific research [1]. According to project requirements, some problems in the construction of safety engineering lab were discussed by analyzing the safety engineering laboratory (SEL) construction problems based on safety engineering laboratory construction practice and combined with safety engineering characteristics.

\section{The constructional fundament and existing problems of SEL}

\section{A The constructional fundament of SEL}

Since 2006, the Chong Qing Safety Engineering Institute (CQSEI) in Chong Qing University of Science \& Technology (CQUST) enrolled full-time students of safety engineering undergraduate specialty. The specialty involved in three professional directions such as petroleum, chemical and mining and has 400students now. Meanwhile, CQSEI has Chongqing safety production research co., LTD which has the national Class-A qualification safety assessment company by the college teachers as the main body. The total amount of evaluation each year is over 5 million.

Since its foundation, CQSEI, by declaring the annual teaching laboratory construction project and provincial professional center laboratory construction projects, strived for more than 6 million laboratory construction funds successively from national related departments, Chongqing and CQUST by the beginning of 2009. Five labs were built in three batches.

(1) Safety ergonomic and emergency rescue lab. The lab, which was built in 2007, includes safety ergonomic teaching experiment and emergency rescue demonstration. The main environments in safety ergonomic lab are instrument of attention distribution, conditioned reflex experiment instrument, dark adapt meter and memory apparatus, etc. The main environments of emergency rescue demonstration are advanced cardiopulmonary resuscitation and traumatic simulation which controlled by computer, the sixth generation advanced cardiopulmonary resuscitation training simulator and Fire protection insulation clothing, etc.

(2) Safety engineering teaching lab. The lab, which was built in 2008, is presented in four modules. The main environments of oil safety module are automatic Martin closed cup flash point tester, Petroleum products flash point tester and lightning and flash point tester, etc. The main environments of mining safety module are gas detector, convergence gauge pressure box, audiovisual material of analysis of coal mine accidents, catastrophic airflow reversal demonstration device, self-rescuer, fire extinguisher and portable instruments, etc. The main environments of chemical safety module are detector formaldehyde, electrical resistance probe corrosion tester, coating thickness gauge and high precision portable digital millimeters. The main environments of 
occupation hazard detection module are digital dust measuring instrument, portable indoor air quality monitor and hygrometer, etc. The main environments of safety evaluation of simulation module are 50 computers and its corresponding network, spark detector pinhole, pipeline locator, laser distance meter and external anticorrosive layer detection instrument on buried pipeline, etc.

(3) Non-mine safety and occupation hazard detection laboratory in Chongqing city. It was built in 2009. The main environments in the lab are acoustic emission detector, Underground pipeline leak detector, Insulation resistance tester, integrated test instrument, differential scanning calorimeter, accident scene survey box, explosion-proof camera, on-site inspection car, determination of basic parameters of complete sets of equipment for dangerous chemicals, system parameter test device of dust explosion and gas, etc. The main environments in the occupation hazard detection and identification lab are respiratory dust sampler, the concentration of dust emission tester, noise spectrum analyzer, multi-channel sound and vibration analyzer, gas chromatograph, atomic fluorescence spectrophotometer, ultraviolet and visible spectrophotometer, atomic absorption spectrophotometer, portable gas chromatograph, sample digestion device, magnetic Stirrers, ultrasonic cleaner, constant temperature water bath box, centrifuge, etc.

\section{$B$ Problems of safety engineering lab construction}

As the construction basic of safety engineering lab, there is clearly a lack of systematic and characteristic prominent problems in the initial construction of safety engineering lab.

(1) The lab pre-construction is lack of overall planning.

Safety ergonomics laboratory, emergency laboratory and safety engineering teaching laboratory were invested by CQUST. The non mining lab and occupation hazard detection laboratory, as the provincial professional center lab of state safety production technical support system, were built by CQSEI. Its construction aim is to provide safety inspection technical support for Chongqing safety supervision departments [2]. Because of the different construction aim, the above three lab construction project actually formed two parts such as teaching laboratory and the foreign technical service lab. Although at a cost of more than 6 million, the above three lab project didn't form the experimental system which adapt to the professional curriculum system of safety engineering. So it was very difficult to become the powerful support for safety engineering discipline development.

(2) Laboratory pr-construction characteristics are not outstanding.

Safety inspection and safety assessment are important skills for students of safety engineering. CQUST has profound foundation of detection and inspection. Meanwhile, CQSEI has Chongqing Yuyou technology co., LTD which has the national class qualification of safety evaluation. From the above three lab project construction, we can see that the safety engineering lab pre-construction did not enhance and highlight these characteristics.

\section{The safety engineering lab construction mode of integrate resources to highlight the characteristics}

The main task of the safety engineering lab construction is lab resources integration, straightening out relations between disciplinary development and laboratory construction, maximizing the laboratory teaching function and improving the education level and prominent characteristic to improve the development of CQSEI.

CQSEI declared and was approved 3.6 million lab construction funds in October, 2009 and have been built and put into use at present. According to project requirements of make full use of existing resources and integrate or newly-built laboratory [1], in the practice of laboratory construction, we put forward the safety engineering laboratory construction mode-integrate resources to highlight the characteristic in order to solve the above problem.

\section{A Integrate resources to form a complete safety engineering experiment system}

According to the characteristics of the safety laboratory pre-construction, the safety engineering lab implementation plan was divided to four big modules based on training plan of safety engineering major.

(1) The first module is safe basic theory practice teaching module. It mainly includes electrical 
safety experiment equipment, mine safety experimental equipment, special equipment teaching experimental equipment and safety experiment equipment in hazardous environment, etc.

(2) The second module is major hazard source detection and monitoring module. It mainly includes carbon sulfur analyzer, DCVG/CIPS, determination of anchor quality and the electrochemical workstation, etc.

(3) The third module is occupation hazard detection module. It mainly includes high performance liquid chromatograph and intelligent Fourier transform infrared spectrometer, etc.

(4) The fourth module is risk prediction and evaluation module. It mainly includes Safeti software, Yantu 5.5, 3D- $\sigma$ and 20 computers, etc.

Among them, the first module was used to strengthen teaching laboratory in 2007 and 2008 to constitute the safety engineering experimental system corresponds to the curriculum system. The second and third modules were used to reinforce the two Chongqing professional laboratory center to increase the teaching function. In this way it could be integrated experiment system [3-5]. The fourth module was used to enhance safety evaluation simulation laboratory and increase characteristics of safety engineering laboratory.

Based on this, safety engineering laboratory was formed by public basic laboratory, professional basic laboratory, professional direction laboratory and professional characteristics laboratory. It was shown in Fig.1.

\section{$B \quad$ Highlighting characteristic-safety inspection and safety evaluation}

In the practice of safety engineering lab construction, the characteristic of safety inspection and safety evaluation was highlighted with professional lab based on the characteristic of safety engineering and superiority of CQSEI.

Safety inspection runs through safety engineering discipline, no one could be out of it no matter what kind of background or direction in safety engineering. The ability cultivation of safety inspection is a significant foundation for students' employment and development in the future majoring in safety engineering. Safety inspection is the key of safety engineering laboratory construction; its investment is more than 2 million. These equipments in lab are mainly used to strengthen two Chongqing professional laboratory center, also they can train the students' inspection capacity of major hazard and occupation hazards in the workplace.

As the important link in risk management, safety evaluation has the profound meaning for improving the safety management level of enterprises, strengthening labor protection and improving the level of safety production. Therefore, our country in the form of legal requirements the related enterprises must be conducted safety evaluation. At the same time, safety assessment is the core curriculum of safety engineering. Many courses such as oil production safety technology and HSE management, chemical safety technology and mining disaster prevention technology all involved in safety evaluation. Safety engineering lab made the purchase of three evaluation software such as SAFETI, Yantu5.5 and 3D- $\sigma$ which could be used in petrochemical industry. Meanwhile, nearly 1 million was invested to buy computers and related video equipment for safety evaluation of simulation laboratory. 


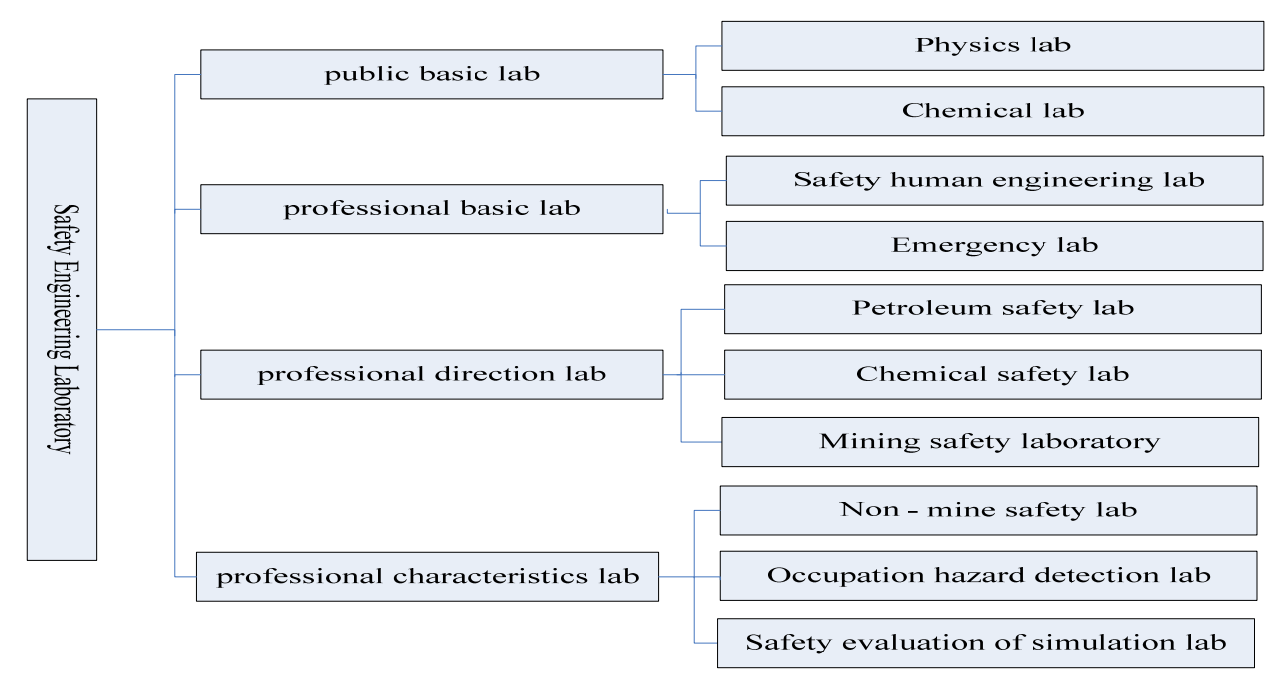

Fig.1.Safety engineering lab structure

\section{Safety engineering laboratory construction practice}

At present, the safety engineering laboratory equipments were basically in place and formed according to the characteristics of integration of resources and outstanding characteristics. It played very good benefits. Professional basic lab, professional basic lab and professional characteristics lab all set related experiment to meet the teaching requirements.

In the aspect of safety inspection, many experiments such as reliability detection experiments on materials and equipment, hazardous chemicals field test, detection experiment of pipeline corrosion, electrical safety testing experiment, identification experiment of dangerous goods, metal and nonmetal analysis experiment, organic and inorganic materials analysis experiment and two week training college were set. In this way the students could master the skills of safety inspection.

In the aspect of safety evaluation, it guided the student to view and practice to increase the understanding and consolidation of knowledge rely on safety Evaluation Company. Meanwhile, the students were directly involved in the specific project in safety evaluation weeks and did it in safety evaluation of simulation laboratory. So it could cultivate the students' proficiency in a particular line to find a way out as they go out into the world. In addition, some teachers in our college are actively using safety evaluation simulation lab resources to apply for research project and doing related research activities.

\section{Conclusions}

The construction mode of integrate resources and highlighting characteristics based on the safety engineering discipline characteristics and combined with the pre-construction actual was put forward. It embodies the teaching as the center and meanwhile highlights characteristics of safety inspection and safety evaluation.

\section{References}

[1] Ministry of finance. Notice on compilation of the laboratory project planning of university characteristic advantage discipline built by the central government worked with local governments in 2007 to 2010, 2007.

[2] State Administration of Work Safety. Construction contract of provincial center for laboratory technical support system for safety production.2008.

[3] Li Quing-yuan and Jiang Hai-ming, A congsideration on implementing the labortory project built by jiont efforts of gental and local authorities [J]. Research and Exploration in Laboratory, 201130 (9) 191-193.

[4] Bao min-qin, Safety management analysis of college laboratory in the new era [J]. Research and Exploration in Laboratory, vol. 30, Nov. 201130 (11) 188-190.

[5] Xiao Ze-long, Wang Jing and Hu Tai-yang, "Reflections on experiment constuction for military industry majors [J]. Research and Exploration in Laboratory, 201130 (10) 190-191. 